\title{
Modified Crossed-Wire Antennas Radiating a Circularly Polarized Conical Beam
}

\author{
Kazuhide Hirose $\mathbb{D}^{1},{ }^{1}$ Kazuhiko Hata, ${ }^{1}$ and Hisamatsu Nakano ${ }^{2}$ \\ ${ }^{1}$ College of Engineering, Shibaura Institute of Technology, Tokyo 135-8548, Japan \\ ${ }^{2}$ Science and Engineering, Hosei University, Tokyo 184-8584, Japan \\ Correspondence should be addressed to Kazuhide Hirose; khirose@sic.shibaura-it.ac.jp
}

Received 18 November 2019; Revised 8 January 2020; Accepted 21 January 2020; Published 24 February 2020

Academic Editor: Shah Nawaz Burokur

Copyright (c) 2020 Kazuhide Hirose et al. This is an open access article distributed under the Creative Commons Attribution License, which permits unrestricted use, distribution, and reproduction in any medium, provided the original work is properly cited.

\begin{abstract}
Two types of modified crossed-wire antennas are investigated to enhance a circularly polarized (CP) wave bandwidth. The wire length of each antenna is increased twice as long as that of the original antenna. First, a bent-type antenna is analyzed using the method of moments. It is found that the $\mathrm{CP}$ wave bandwidth for a $3 \mathrm{~dB}$ axial ratio criterion is twice as wide as that of the original antenna. Next, a spiral-type antenna is analyzed. It is revealed that the antenna shows a CP wave bandwidth of $28 \%$, which is wider than that of the original antenna by a factor of 3.5. The analysis results are validated by experimental work.
\end{abstract}

\section{Introduction}

Mobile satellite communications have often required an antenna radiating a circularly polarized $(\mathrm{CP})$ conical beam [1]. For this requirement, many antennas have been investigated [2-5]. These antennas are mainly divided into two classes: planar $[2,3]$ and nonplanar $[4,5]$. In addition, there have been three kinds of radiators used in the nonplanar antennas; they are an aperture [5], slot [6], and monopole [4].

A crossed-wire antenna shown in Figure 1(a)is in a class with the nonplanar antennas having a monopole radiator [7]. The antenna has an advantage over a conventional one [2-6] in that the maximum radiation can be obtained at a lower elevation-angle direction. So far, there has been no investigation to increase the $\mathrm{CP}$ wave bandwidth.

The purpose of this paper is to increase the $\mathrm{CP}$ wave bandwidth of a crossed-wire antenna. For this, two types of antennas with increased wire length are analyzed using the method of moments [8]; one is a bent-type antenna shown in Figure 1(b) and the other is a spiral-type antenna shown in Figure 1(e), each having wire $o-o^{\prime}$ shown Figure 1(c) as a monopole radiator. The radiation characteristics including the axial ratio, beam direction, input impedance, and radiation pattern are discussed and compared with those of the original antenna [7] shown in Figure 1(d).

\section{Bent-Type Antenna}

In this section, the arm length of the original antenna [7] shown in Figure 1(d) is increased. The effects of the increased arm length on the radiation characteristics are investigated.

Figures $1(a)-1(c)$ illustrate a bent-type antenna, which is made of wires of radius $\rho$. Four bent arms symmetrical with respect to the cross point $o^{\prime}$ are backed by a ground plane at height $h$. The arms are fed at the cross point $o^{\prime}$ through a vertical wire $o^{\prime}-o$ of length $h$, as shown in Figure 1(c), and the bottom end $o$ is excited by a coaxial line. To obtain CP radiation, four parasitic elements of length $h$ and radius $\rho$ are added symmetrically with respect to the cross point $o^{\prime}$ [7]. The location $(x, y)$ of the parasitic element is designated as $\left(d_{x}, d_{y}\right)$, as shown in Figure 1(b). Note that the bottom end of each parasitic element is short-circuited to the ground plane.

The antenna is designed to radiate a CP wave in the maximum beam direction. For this, the parasitic element location $\left(d_{x}, d_{y}\right)$ is appropriately selected [7] using the method of moments [8]. To enhance the CP wave 


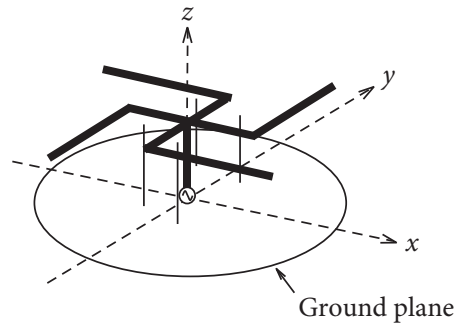

(a)

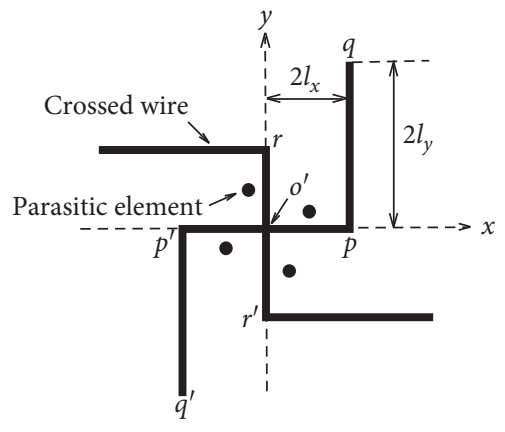

(b)

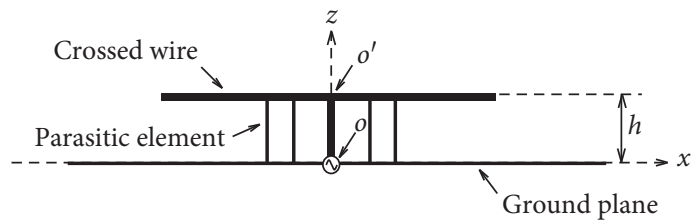

(c)

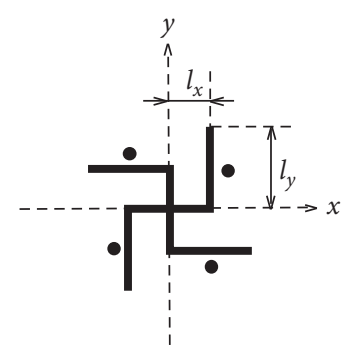

(d)

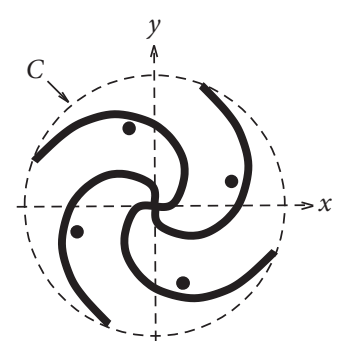

(e)

Figure 1: Antenna configurations. (a) Perspective view. (b) Top view of bent-type antenna. (c) Side view. (d) Original antenna [7]. (e) Spiraltype antenna.

bandwidth, the bent arm length is taken to be twice $\left(2 l_{x}+2 l_{y}\right)$ as long as that $\left(l_{x}+l_{y}=3 \lambda_{0} / 8\right)$ of the original antenna, as shown in Figures 1(b) and 1(d), where $l_{x}$ and $l_{y}$ are element lengths in the $x$ and $y$ directions of the bent arm in the first quadrant, respectively, and $\lambda_{0}$ is the free-space wavelength at a test frequency of $f_{0}$. The other configuration parameters are chosen to be the same as those of the original antenna [7]: ( $h$, $\left.\rho, l_{x}, l_{y}\right)=\left(\lambda_{0} / 4, \lambda_{0} / 85, \lambda_{0} / 8, \lambda_{0} / 4\right)$. Note that the ground plane is assumed to be of an infinite extent and image theory is used in the analysis [7].

A numerical result of the axial ratio versus frequency for $\left(d_{x}, d_{y}\right)=\left(2 \lambda_{0} / 32,4 \lambda_{0} / 32\right)$ is shown with a solid line in Figure 2(a). The axial ratio is evaluated in the maximum beam direction. It is found that the antenna has a $\mathrm{CP}$ wave bandwidth of $15 \%$ for a $3 \mathrm{~dB}$ axial ratio criterion. For comparison, a dotted line shows the result of the original antenna, which has a bandwidth of $8 \%$ [7]. The bandwidth of the bent-type antenna is twice as wide as that of the original antenna. A solid line of Figure 2(b) shows the beam direction $\theta$ versus frequency, where the direction remains almost unchanged (at $\theta=53^{\circ} \pm 2.5^{\circ}$ ) in the $\mathrm{CP}$ wave bandwidth.

The input impedance as a function of frequency is shown with solid lines in Figure 2(c). It is observed that the real part of the impedance $R_{\text {in }}$ reaches $1300 \Omega$ at a lower frequency in the $\mathrm{CP}$ wave bandwidth.

Finally, we explain why the antenna can radiate a CP wave when the parasitic element location $\left(d_{x}, d_{y}\right)$ is selected. For simplicity, we consider radiation components of $E_{\theta}$ and $E_{\phi}$ in the $\phi=0^{\circ}$ plane. Referring to the antenna coordinate system shown in Figure 1, we see that partial radiation from the parasitic elements has only the $E_{\theta}$ component. On the other hand, partial radiation from both the four bent arms and vertical wire $o^{\prime}-o$ has not only the $E_{\theta}$ component but also the $E_{\phi}$ component since each arm is bent. To be more specific, the $E_{\phi}$ component occurs from wires $p-q$ and $p^{\prime}-q^{\prime}$ in the bent arms shown in Figure 1(b), since partial radiation from the wire $o^{\prime}-r$ cancels out that from the wire $o^{\prime}-r^{\prime}$ due to their current distributions having the same amplitudes with a phase difference of $180^{\circ}$ (this implies a fact that if the four arms were straight, the antenna could not radiate a CP wave even when the parasitic element location is selected). The selection of $\left(d_{x}, d_{y}\right)$ leads to the $E_{\theta}$ component appropriate for $\mathrm{CP}$ radiation. It can be said that appropriate radiation of the $E_{\theta}$ component by the selection of $\left(d_{x}, d_{y}\right)$ enables the antenna to radiate a $\mathrm{CP}$ wave.

The abovementioned explanation suggests a method for increasing the CP wave bandwidth. Since the selection of $\left(d_{x}, d_{y}\right)$ contributes to the control of the $E_{\theta}$ component, it is necessary to control the $E_{\phi}$ component. We can control the $E_{\phi}$ component by increasing the arm length and/or by changing the arm shape. From this point of view, we first increase the arm length in this section and then change the bent arms into spiral ones in the next section.

\section{Spiral-Type Antenna}

So far, we have investigated the effects of an increased arm length on the radiation characteristics of a bent-type antenna. In this section, a bent arm with an increased length is further transformed into a spiral one shown in Figure 1(e). The effects of the spiral arm on the radiation characteristics are investigated.

Figure 3 shows a spiral-type antenna of circumference $C$, where the arms are symmetrical with respect to the cross point $o^{\prime}$. Each arm $\# n(n=1,2,3$, and 4$)$ is defined by an equiangular spiral function with a spiral constant $a_{\mathrm{s}}$ and an 


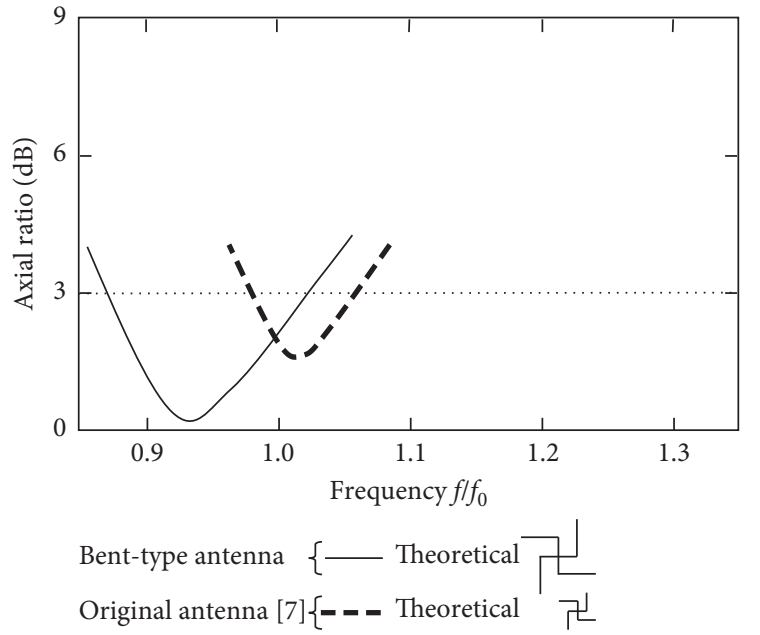

(a)

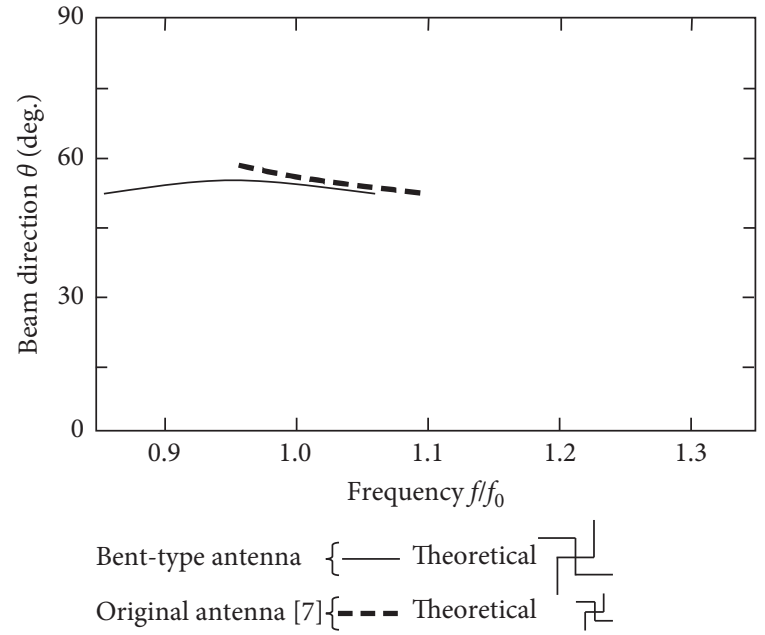

(b)

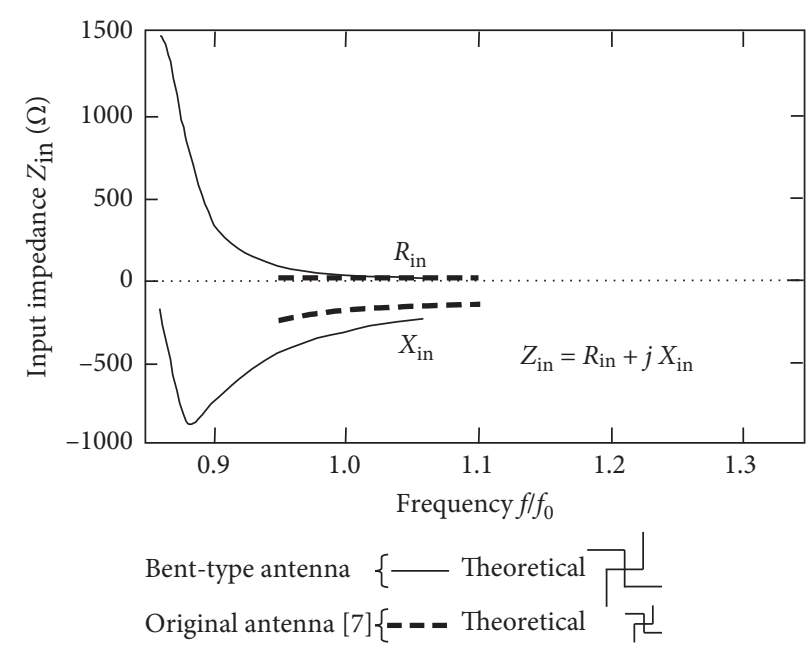

(c)

FIgURE 2: Frequency responses of axial ratio, beam direction, and input impedance of bent-type antenna for $\left(d_{x}, d_{y}\right)=\left(2 \lambda_{0} / 32,4 \lambda_{0} / 32\right)$, together with those of the original antenna [7]. (a) Axial ratio. (b) Beam direction. (c) Input impedance.

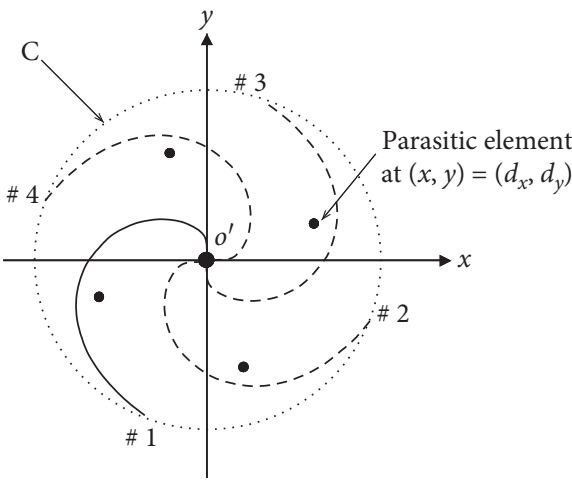

(a)

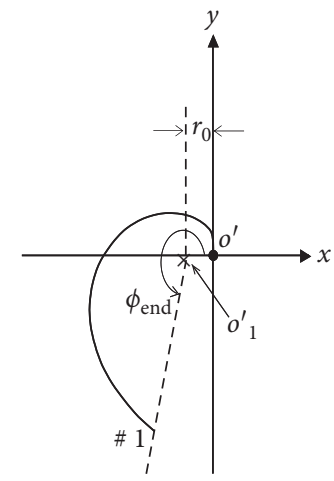

(b)

Figure 3: Spiral-type antenna. (a) Arms \#n $\left(n=1,2,3\right.$, and 4) with cross point $o^{\prime}$. (b) Spiral origin $o_{n}^{\prime}$. Arm shape is defined by the equiangular spiral function: $r=r_{0} \cdot \exp \left[a_{s} \cdot\left\{\phi_{s}-(n-1) \cdot \pi / 2\right\}\right]$, where $r$ is the radial distance from the spiral origin $o_{n}^{\prime}$ to a point on the spiral arm $\# n, r_{0}$ is the initial length of $r, a_{\mathrm{s}}$ is a spiral constant, and $\phi_{\mathrm{s}}$ is a winding angle ranging $(n-1) \cdot \pi / 2 \leq \phi_{\mathrm{s}} \leq(n-1) \cdot \pi / 2+\phi_{\mathrm{end}}$ with $\phi_{\mathrm{end}}$ being the end winding angle. 


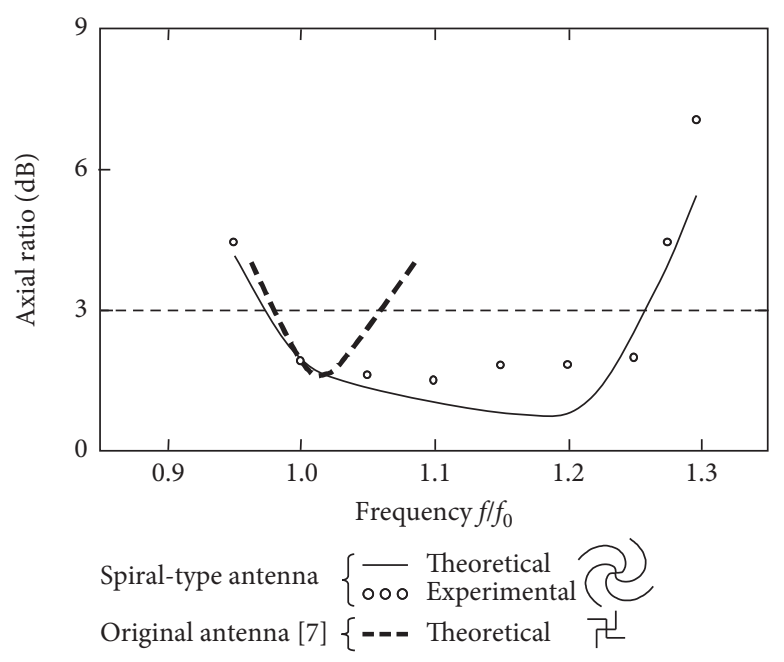

(a)

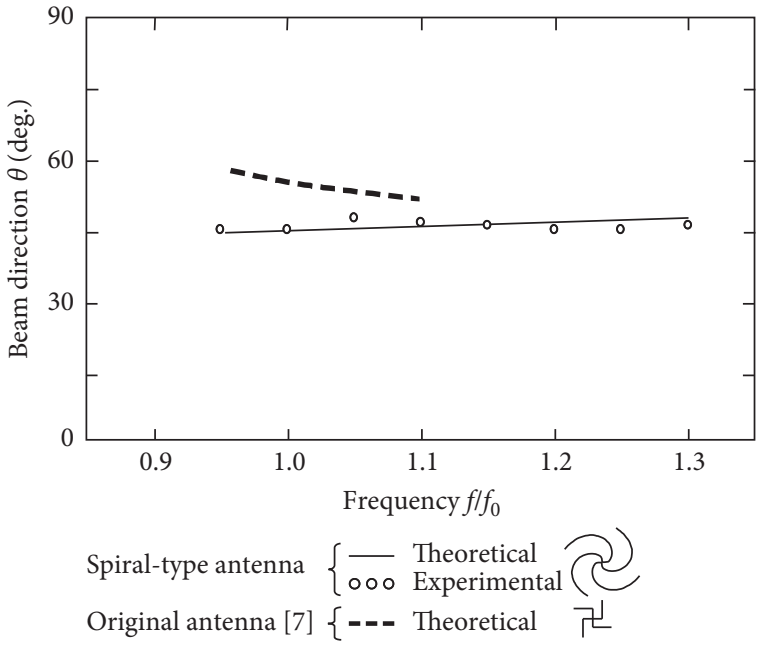

(b)

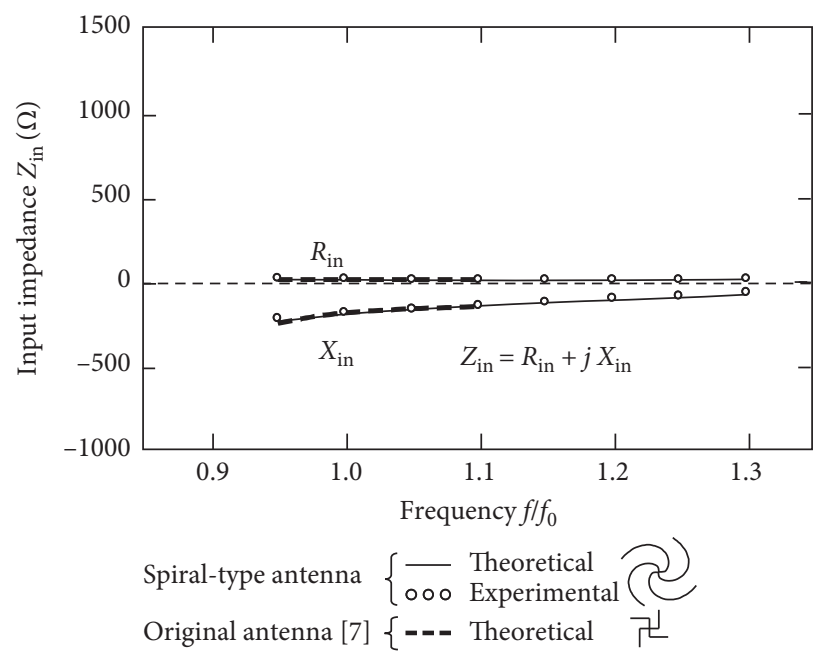

(c)

Figure 4: Frequency responses of axial ratio, beam direction, and input impedance of a spiral-type antenna for $\left(d_{x}, d_{y}\right)=\left(57 \lambda_{0} / 256,15 \lambda_{0} /\right.$ 256), together with those of the original antenna [7]. (a) Axial ratio. (b) Beam direction. (c) Input impedance.

end winding angle $\phi_{\text {end. }}$. Note that the spiral origin $o_{n}^{\prime}$ is shifted from the cross point $o^{\prime}$ by a distance of $r_{0}$, as shown in Figure 3(b).

The antenna circumference is chosen to be a typical value $\left(C=2.4 \lambda_{0}\right)$ for a conventional spiral antenna to radiate a $\mathrm{CP}$ conical beam [9]. The arm length is taken to be the same as that $\left(3 \lambda_{0} / 4\right)$ in section 2 . From these two values, the two spiral parameters are determined to be $\left(a_{\mathrm{s}}, \phi_{\mathrm{end}}\right)=\left(0.5 \mathrm{rad}^{-1}\right.$, $4.5 \mathrm{rad}$ ) for a distance of $r_{0}=0.04 \lambda_{0}$. The other configuration parameters are the same as those in section 2, except for the parasitic element location $\left(d_{x}, d_{y}\right)$.

The location $\left(d_{x}, d_{y}\right)$ is again optimized for CP radiation. The numerical result of the axial ratio versus frequency for $\left(d_{x}, d_{y}\right)=\left(57 \lambda_{0} / 256,15 \lambda_{0} / 256\right)$ is shown with a solid line in Figure $4(\mathrm{a})$, together with that of the original antenna. It is emphasized that the antenna shows a CP wave bandwidth of $28 \%$, which is wider than that of the original antenna by a factor of 3.5.

A solid line in Figure 4(b) shows the frequency response of the maximum beam direction. It is observed that the direction is almost constant $\left(\theta=46^{\circ} \pm 1^{\circ}\right)$ in the CP wave bandwidth. In fact, the slight variation is half that of $\left( \pm 2.5^{\circ}\right)$ the bent-type antenna in section 2 . It can be said that the spiral arm contributes to not only enhancement in the $\mathrm{CP}$ wave bandwidth but also to stabilization of the beam direction.

Stabilization in the input impedance versus frequency is also obtained. This is shown with solid lines in Figure 4(c). It is found that the real part of the impedance remains almost unchanged at $R_{\text {in }}=14 \Omega$ in the CP wave bandwidth (while the $R_{\text {in }}$ of the bent-type antenna reaches $1300 \Omega$ in section 2 ). Note that the input impedance of the original antenna is also shown with dotted lines, which are not visible as they overlap the solid lines.

Figures 5(a) and 5(b) show typical radiation patterns in the $\phi=0^{\circ}$ and $45^{\circ}$ planes, respectively, for $\left(d_{x}, d_{y}\right)=\left(57 \lambda_{0} /\right.$ $\left.256,15 \lambda_{0} / 256\right)$. The pattern in the $\phi=90^{\circ}$ plane is the same as that in the $\phi=0^{\circ}$ plane due to the symmetrical antenna configuration with respect to the coordinate origin, $o$. Solid and dotted lines show the numerical results of left-hand $\left(E_{\mathrm{L}}\right)$ 


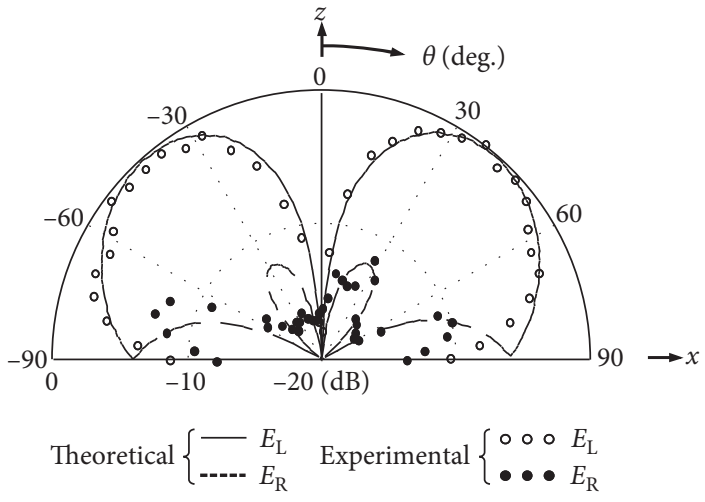

(a)

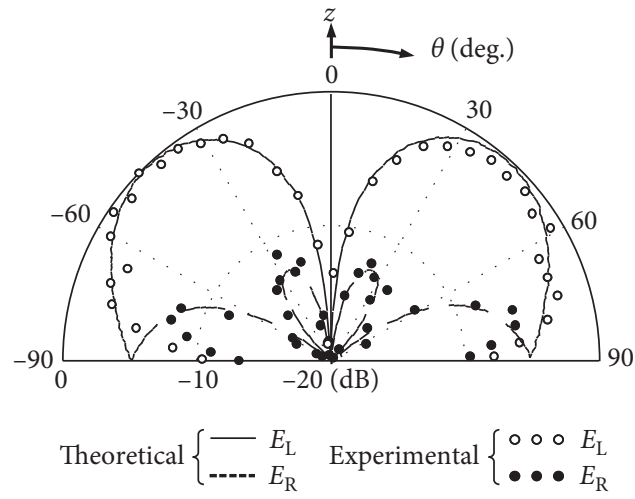

(b)

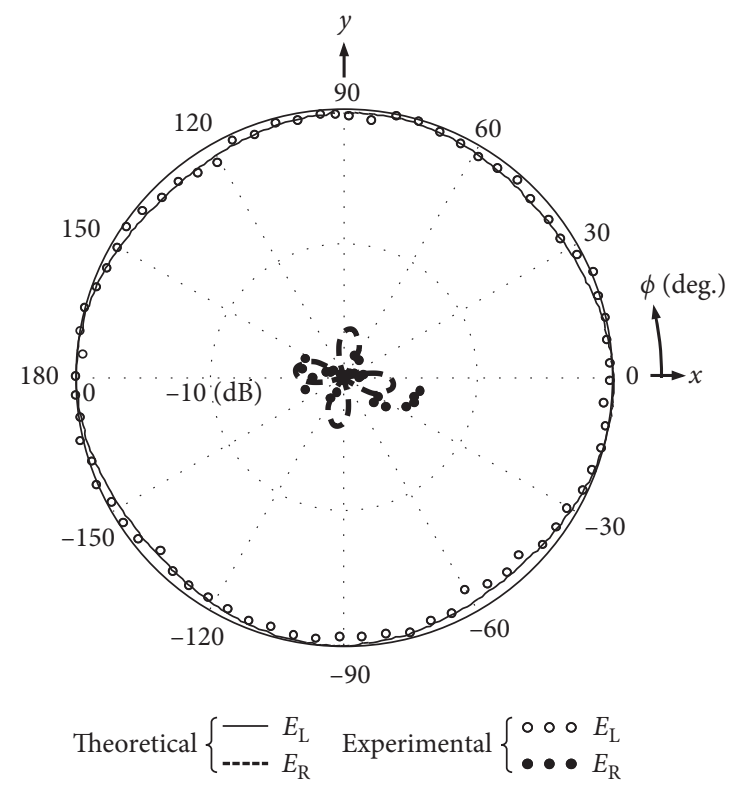

(c)

Figure 5: Radiation patterns of spiral-type antenna for $\left(d_{x}, d_{y}\right)=\left(57 \lambda_{0} / 256,15 \lambda_{0} / 256\right)$ at $f=1.0 f_{0}$. (a) $\phi=0^{\circ}$ plane, where the beam direction is $\theta=46^{\circ}$. (b) $\phi=45^{\circ}$ plane. (c) $\theta=46^{\circ}$ plane.

and right-hand $\left(E_{\mathrm{R}}\right) \mathrm{CP}$ wave components, respectively. It is observed that the antenna radiates a CP conical beam. The maximum beam directions are $\theta=46^{\circ}$ and $44^{\circ}$ in the $\phi=0^{\circ}$ and $45^{\circ}$ planes, respectively. Angle coverages where the axial ratio is less than $3 \mathrm{~dB}$ are $20^{\circ}$ and $18^{\circ}$ in the $\phi=0^{\circ}$ and $45^{\circ}$ planes, respectively. The gain is approximately $5 \mathrm{~dB}$.

Solid and dotted lines in Figure 5(c) show the numerical result of the radiation pattern in the $\theta=46^{\circ}$ plane, where the maximum radiation is obtained. It is found that the principal polarization component $E_{\mathrm{L}}$ remains almost unchanged versus angle $\phi$. The variation in $E_{\mathrm{L}}$ is within $0.8 \mathrm{~dB}$.

\section{Experimental Work}

In this section, the numerical results in section 3 are validated by experimental work. For this, we fabricate a spiraltype antenna using the ground plane of $12 \lambda_{0} \times 12 \lambda_{0}$ at $f_{0}=7 \mathrm{GHz}$. Pictures of the fabricated antenna are shown in Figure 6 . The radiation patterns are measured in an anechoic chamber using a horn antenna (Narda 642) at a distance of $3 \mathrm{~m}$ from the test antenna. The test antenna is rotated on a turntable, while the horn antenna is fixed. The input impedance is measured using a network analyzer (HP 8719C).

Small circles in Figure 4 show the experimental results of the frequency responses of the axial ratio, beam direction, and input impedance. They agree well with the numerical results shown with solid lines.

Agreement between the experimental and numerical results is also obtained for the radiation patterns shown in Figure 5. Small circles and dots show the experimental results of principal $\left(E_{\mathrm{L}}\right)$ and cross polarization $\left(E_{\mathrm{R}}\right)$ components, respectively. The corresponding numerical results are shown with solid and dotted lines.

Before concluding the investigation of modified crossedwire antennas, we compare the results with those of existing nonplanar antennas. The comparison is summarized in Table 1. It is emphasized that the spiral-type antenna (prototype) has a wider CP wave bandwidth than the other 


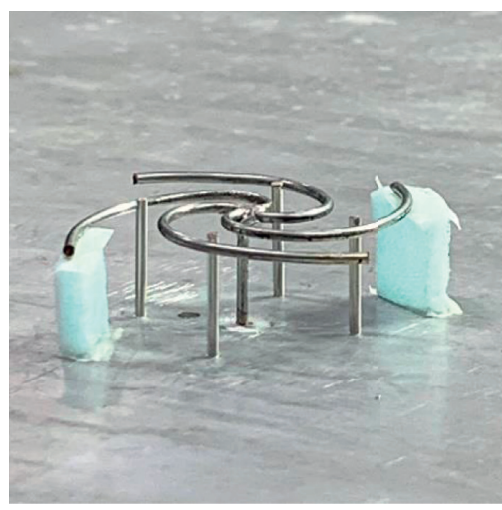

(a)

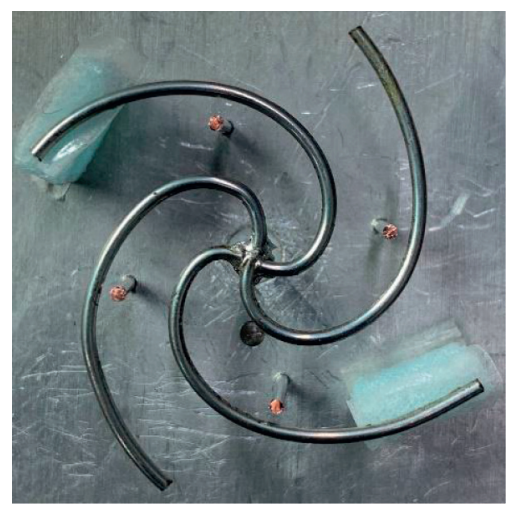

(b)

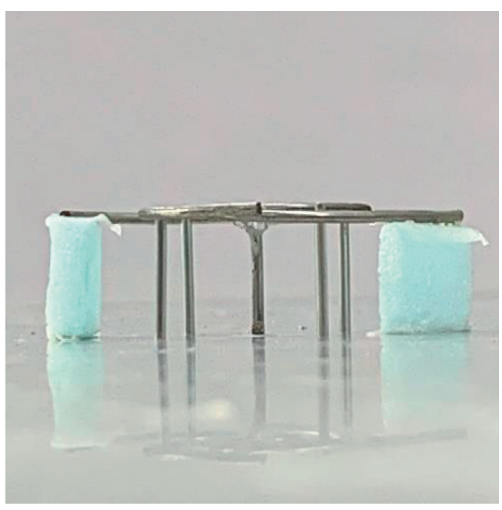

(c)

Figure 6: Pictures of the prototype used in the experimental work. (a) Perspective view. (b) Top view. (c) Side view.

TABLE 1: Comparison with other nonplanar antennas.

\begin{tabular}{lccc}
\hline Radiator in antenna & CP wave bandwidth (\%) & Profile $(x \times y \times$ height $)$ & Beam direction from zenith $\left(^{\circ}\right)$ \\
\hline Aperture [5] & 5 & $1.6 \lambda \times 1.6 \lambda \times 1.0 \lambda$ & 28 \\
Slot [6] & 9 & $2.5 \lambda \times 2.5 \lambda \times 1.0 \lambda$ & 45 \\
Monopole [4] & 22 & $1.0 \lambda \times 1.0 \lambda \times 0.35 \lambda$ & 35 \\
Monopole [7] & 8 & $0.50 \lambda \times 0.50 \lambda \times 0.25 \lambda$ & 55 \\
Monopole [10] & $3^{*}$ & $0.48 \lambda \times 0.48 \lambda \times 0.45 \lambda$ & 50 \\
Monopole (prototype) & 28 & $0.76 \lambda \times 0.76 \lambda \times 0.25 \lambda$ & 46 \\
\hline
\end{tabular}

${ }^{*} 3 \%$ is the bandwidth for VSWR $<1.5$.

antennas. In addition, the prototype with increased arm length still has a small antenna size and a large-angle beam direction from the zenith (a low elevation-angle direction) in comparison with the other antennas.

\section{Conclusions}

Using the method of moments, we have investigated a crossed-wire antenna modified for wideband circularly polarized (CP) radiation. First, the bent arm length of the original antenna is increased. It is found that the CP wave bandwidth becomes twice that of the original antenna. Subsequently, the bent arm with an increased length is transformed into a spiral one. It is demonstrated both numerically and experimentally that the antenna shows a CP wave bandwidth of $28 \%$, which is 3.5 times as wide as that of the original antenna.

Input impedance matching a $50 \mathrm{ohm}$ coaxial line has yet to be researched.

\section{Data Availability}

The numerical and experimental data used to support the findings of this study are included within the article.

\section{Conflicts of Interest}

The authors declare that there are no conflicts of interest regarding the publication of this paper.

\section{Acknowledgments}

The authors would like to thank Blair Thomson for his invaluable assistance in the preparation of this manuscript.

\section{References}

[1] J. R. James and P. S. Hall, Handbook of Microstrip Antennas, Peregrinus, Stevenage, U.K, 1989.

[2] H. Xu, J. Zhou, Q. Wu, Z. Yu, and W. Hong, "Wideband lowprofile SIW cavity-backed circularly polarized antenna with high-gain and conical-beam radiation," IEEE Transactions on Antennas and Propagation, vol. 66, no. 3, pp. 1179-1188, 2018.

[3] W. Lin and H. Wong, "Circularly polarized conical-beam antenna with wide bandwidth and low profile," IEEE Transactions on Antennas and Propagation, vol. 62, no. 12, pp. 5974-5982, 2014.

[4] S. Karki, M. Sabbadini, K. Alkhalifeh, and C. Craeye, "Metallic monopole parasitic antenna with circularly polarized conical patterns," IEEE Transactions on Antennas and Propagation, vol. 67, no. 8, pp. 5243-5252, 2019.

[5] S.-S. Qi, W. Wu, and D.-G. Fang, "Singly-fed circularly polarized circular aperture antenna with conical beam," IEEE Transactions on Antennas and Propagation, vol. 61, no. 6, pp. 3345-3349, 2013.

[6] G. Chenhu, J. Geng, H. Zhou et al., "Truncated circular cone slot antenna array that radiates a circularly polarized conical beam," IEEE Antennas and Wireless Propagation Letters, vol. 16, pp. 2574-2577, 2017.

[7] K. Hirose and T. Wada, "A bent, crossed wire antenna with vertical parasitic elements:formation of a circularly polarized 
conical beam," IEICE Transactions,vol. J84-B, no. 9, pp. 1164-1672, 2001.

[8] R. F. Harrington, Fields Computation by Moment Methods, Macmillan, New York, NY, USA, 1968.

[9] K. Hirose, H. Sato, and H. Nakano, "Spiral antennas radiating a circularly polarized conical beam," in Proceedings of IEEE Antennas and Propagation Society International Symposium, pp. 602-605, Washington, DC, USA, 2005.

[10] H. Kawakami, G. Sato, and R. Wakabayashi, "Research on circularly polarized conical-beam antennas," IEEE Antennas and Propagation Magazine, vol. 39, no. 3, pp. 27-39, 1997. 